\title{
SYNCHRONIZATION OF TRAFFIC LIGHTS FOR HIGH FLOW*
}

\author{
BY \\ G. F. NEWEILL \\ Brown University
}

\begin{abstract}
It is assumed here that the flow of traffic is so heavy that it can be described qualitatively by existing theories wherein each car follows another with no passing and that platoons formed by traffic lights spread so much between lights that the time between the arrival of the first and last cars of a platoon at a light exceeds the green period. Under such conditions it is shown that the best pairwise synchronization of lights gives the best overall synchronization for one-directional traffic. The synchronization which minimizes the total delay for all cars is one for which the tail car of a platoon suffers no delay but the lead car is stopped. For two-directional traffic, heavy in both directions, the best synchronization is very likely to be that which is optimal for the direction of traffic with the lower flow.
\end{abstract}

Introduction. It is an unfortunate fact that in many urban areas sequences of traffic lights are synchronized to minimize the delay for a driver traveling at the local speed limit. This usually works well when the traffic density is low but not during rush hours when the optimal synchronization is needed most and no one is able to maintain the speed for which the lights are timed.

In a previous paper [1] some theory was described relating to the synchronization of lights when the volume of traffic was so low that interactions between cars could be neglected. Here we consider the opposite case where the traffic is dense and the flow through the intersections near saturation. Under such conditions it is reasonable to assume that passing is rare and that cars behave approximately as described by various continuum [2,3] or car-following theories [4-7].

The object is only to describe a few qualitative conclusions. The general problem of how one should synchronize traffic lights is difficult for several reasons. First, very little is known about the dynamics of traffic flow. The only reasonably satisfactory theories proposed so far are those in which the effects of passing can be neglected either because the density is so low that cars seldom interact or the density is so high that there are few opportunities to pass. Secondly the effects of traffic lights on even simple flow patterns can be complicated because generally the pattern of arrivals of cars at one light depend not only upon the synchronization of the nearest previous light but the second, third, etc. lights as well. Finally in any real two dimensional network of roads one can not synchronize the lights on one highway independent of the others since the optimal synchronization for the network may not be the optimal for any given highway.

Here we avoid the first problem by assuming that the density is very high and we avoid the last problem by disregarding the cross-traffic. We shall see that the second problem disappears if the flow is high enough and the lights not too close together. The delays suffered at one light then depend only on the synchronization of the nearest previous light but are independent of lights further away. Thus the optimal synchronization of a long series of lights can be determined by a successive synchronization of con-

*Received April 8, 1963. This research was supported by a grant from General Motors Corporation* 
secutive pairs. This is particularly convenient when lights are not equally spaced along the highway since otherwise the optimal synchronization of one pair of lights would depend not only upon the distance between this pair of lights but also upon the distance and synchronization between the other lights.

In the next section we shall describe some qualitative properties of one-directional traffic through a sequence of lights as predicted by existing car-following theories and identify the conditions necessary to guarantee that the delays suffered at any light depend only upon the synchronization of the previous light. We next consider what synchronization will minimize the total delay under these conditions. If the platoons approaching a light have spread so much in traveling from one light to the next that the time interval between the first and last cars exceeds the green time of the light then some car is certain to be delayed. We conclude that the total delay to all cars is least if the last car arrives at the light just before the light turns red and suffers no delay but the lead car arrives too early and is stopped. This has less delay, for example, than the commonly used synchronization in which the lead car arrives just as the light turns green but the last car consequently arrives after the light turns red.

No attempt is made to evaluate the optimal phase of lights in terms of the velocities of the drivers, spacings, etc. In practice one can observe directly some average arrival times of last cars in platoons and then synchronize the lights so they turn red immediately afterwards. From an experimental point of view it would require less effort to determine the synchronization directly from observed arrival times than to record the velocities of the drivers, distances between lights, etc. which one would need to know in order to compute the arrival times.

Finally we consider the corresponding problem for two directional traffic heavy in both opposing directions. The conditions for optimal synchronization in this case is complicated, however, and is sensitive to certain unrealistic details of the model. The model suggests, however, that in most cases the optimal synchronization will be nearly that for the direction of traffic with the lower flow, contrary to what one might expect. This is true only if the traffic is sufficiently heavy in both directions, however.

The model. Suppose we have a source of traffic approaching the first of a sequence of traffic lights. This is represented at the bottom of Fig. 1 by the series of solid line space-time trajectories of cars. The traffic light at $x=0$ and the red periods are indicated by the heavy horizontal line segments. For simplicity, we assume that the flow $q$, the average number of arrivals per unit time, approaching the first light is less than the capacity of the intersection and if $T$ is the cycle time of the light, that $q T=n$ is integer. The latter assumption is not essential but if it were not true, the flow would be more complicated in detail because the number of departures in each light cycle would have to oscillate between integer values on either side of $q T$ in such a way as to give the average $q T$ over many cycles.

Present theories of traffic flow at high density predict the following behavior. As cars approach the first intersection during a red period, a deceleration shock moves back into the stream indicating the rear of the queue. When the light turns green a fan of acceleration waves shown in Fig. 1 by the broken lines is formed. As these waves move back to the shock, the shock gains forward speed, eventually changes direction, and passes through the intersection. If the flow approaching the intersection is less than the capacity of the intersection, this shock will pass the intersection during the green period as shown in Fig. 1. Cars which arrive after the shock has passed the intersection will 


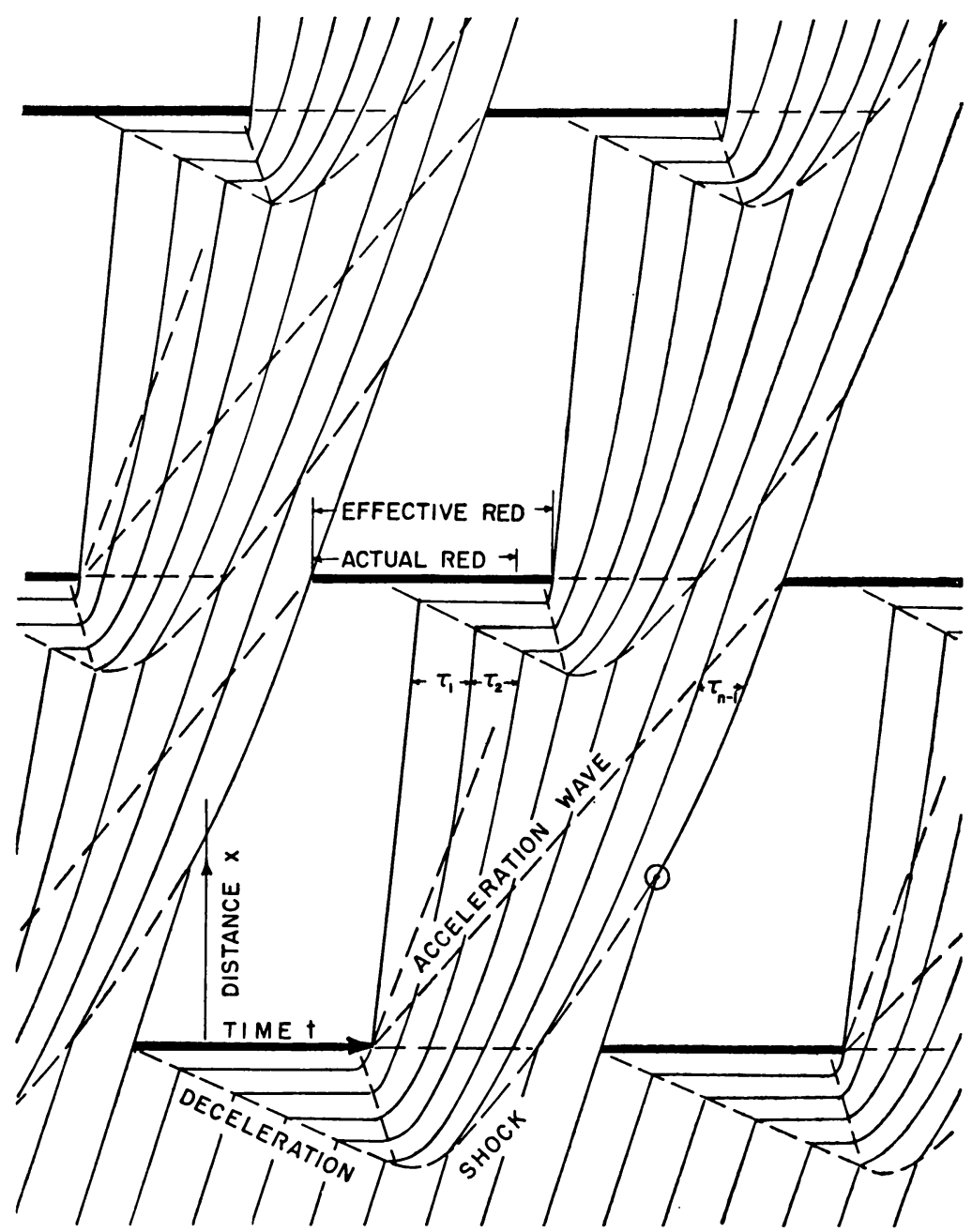

Fig. 1.

continue to overtake the shock beyond the intersection until the flow is cut off by the next red light (the circled point of Fig. 1).

This part of Fig. 1 has been discussed previously in terms of the continuum theory by Lighthill and Whitham [2].

Cars leave the first intersection in platoons of length $n$. The time interval between the first and last cars of a platoon as it passes a point $x$, which we shall call the duration of the platoon, is less than the effective green period $G$ of the light as it passes the first intersection but increases as it moves from one intersection to the next. Part of this increase may be due to differences in the desired speeds of the first few cars in the queue. For example, the lead car may accelerate to a higher speed than the second driver is willing to travel, causing a growing gap to form in the stream. For long platoons, however, (ten cars, for example) the spreading of the platoon is mainly due to the acceleration of the platoon as a whole rather than random differences in speeds of the first few cars.

According to existing theories, the velocities of the cars as they pass the intersection 
after the lights turns green should quickly adjust to a value which yields the maximum possible instantaneous flow at the intersection. Cars continue to accelerate beyond the intersection, however, as they seek the velocity of the lead car which is traveling at its free speed. Since the flow is optimal at the intersection, any increase in velocity gives a lower flow, thus longer time headways and a longer duration of the platoon.

We shall now assume that the flow and distance between intersections are sufficiently large that (a) the duration of the platoon as it approaches a light is longer than $G$, and (b) the circled point of Fig. 1 where the end of the platoon from the first light overtakes the shock wave from the queue at this light occurs between the first and second intersections (not beyond the second). The immediate consequence of $(a)$ is that some car is stopped at every light regardless of synchronization. Assumption (b) guarantees that the arrival pattern at the second light depends only upon the phase of the first light but is independent of the arrival pattern at the first light thus also of the phase of any lights that might have preceded the first. This is so because the velocities of cars beyond the circled point are determined entirely by the fan of acceleration waves originating from the previous light. The deceleration shock cuts off any waves from points further back. With these assumptions one need only consider the synchronization of consecutive pairs of lights to determine the best synchronization of a long sequence of lights.

Optimal synchronization. One possible objective in synchronizing lights is to minimize the total delay for all cars traveling in one direction. We wish to show that optimal synchronization for the type of flow described above is the one shown in Fig. 1 where the last car in the platoon barely clears the green light but the cars at the front of the platoon are stopped. We show this first for the case in which the lead car of the platoon travels at its free speed and the velocities of all cars between lights are given by some rule which does not depend upon the synchronization, i.e. the trajectories of Fig. 1 before they encounter the deceleration shock from the second light are independent of the phase of the second light. We will then show that any other flow pattern in which the lead car travels slower than his free speed gives longer delays.

We are interested only in comparing the delays for various synchronizations and so we will evaluate all delays relative to those shown in Fig. 1 taken as zero. We let $t$ represent the time by which the phase of the second light is advanced relative to the phase of Fig. 1. For a platoon of cars approaching the second light let $D(t)$, the (relative) total delay per light cycle at the second light, be the change in the sum of the arrival times of these cars at the third light due to any change in phase of the second light.

As a result of the assumptions made above that (1) the circled point of Fig. 1 occurs between the first and second lights and (2) the velocities of cars do not depend upon the synchronization, it follows that the time intervals $\tau_{1}, \tau_{2}, \cdots \tau_{n-1}$ between arrivals at a light are independent of the synchronization. If we were to retard the second light by a small amount $|t|, t<0$, the arrival pattern at the third light would be unchanged except that all cars arrive later by a time $|t|$. Thus

$$
D(t)=-n t=n|t|>0 \text { for } t<0
$$

at least for $|t|$ small enough so that the light is not still green when the first car from the next platoon arrives at the second light.

If we advance the second light by an arbitrarily small amount from that of Fig. 1, the last car of the approaching platoon is stopped and it will become the first car to leave in the next light cycle. Correspondingly, there must also be a car waiting from the 
previous light cycle that retards the first car of our approaching platoon. This essentially. zero advance of the light does not change the times of arrivals of cars at the third light but does change the labeling of the cars so that the lead car in the approaching platoon at the second light becomes the second car of the platoon approaching the third light. The change in the sum of arrival times of the original platoon at the third light is one cycle time $T$ because we are adding the arrival times of the second, third, etc. cars at the third light plus that of the first car in the next cycle. Only this last term is different than before and it is $T$ larger. Thus

$$
D(0+)=T
$$

A further advance in the light by an amount $0<t<\tau_{n-1}$ will now reduce $D(t)$ by. an amount $n t$ until for $t=\tau_{n-1}$ we have the flow pattern shown in Fig. 2 where the $(n-1)$ th car of the approaching platoon barely clears the light. We lost a time $T$ because we cut off the last car of the platoon but we recovered a total time $n \tau_{n-1}$ by a shift

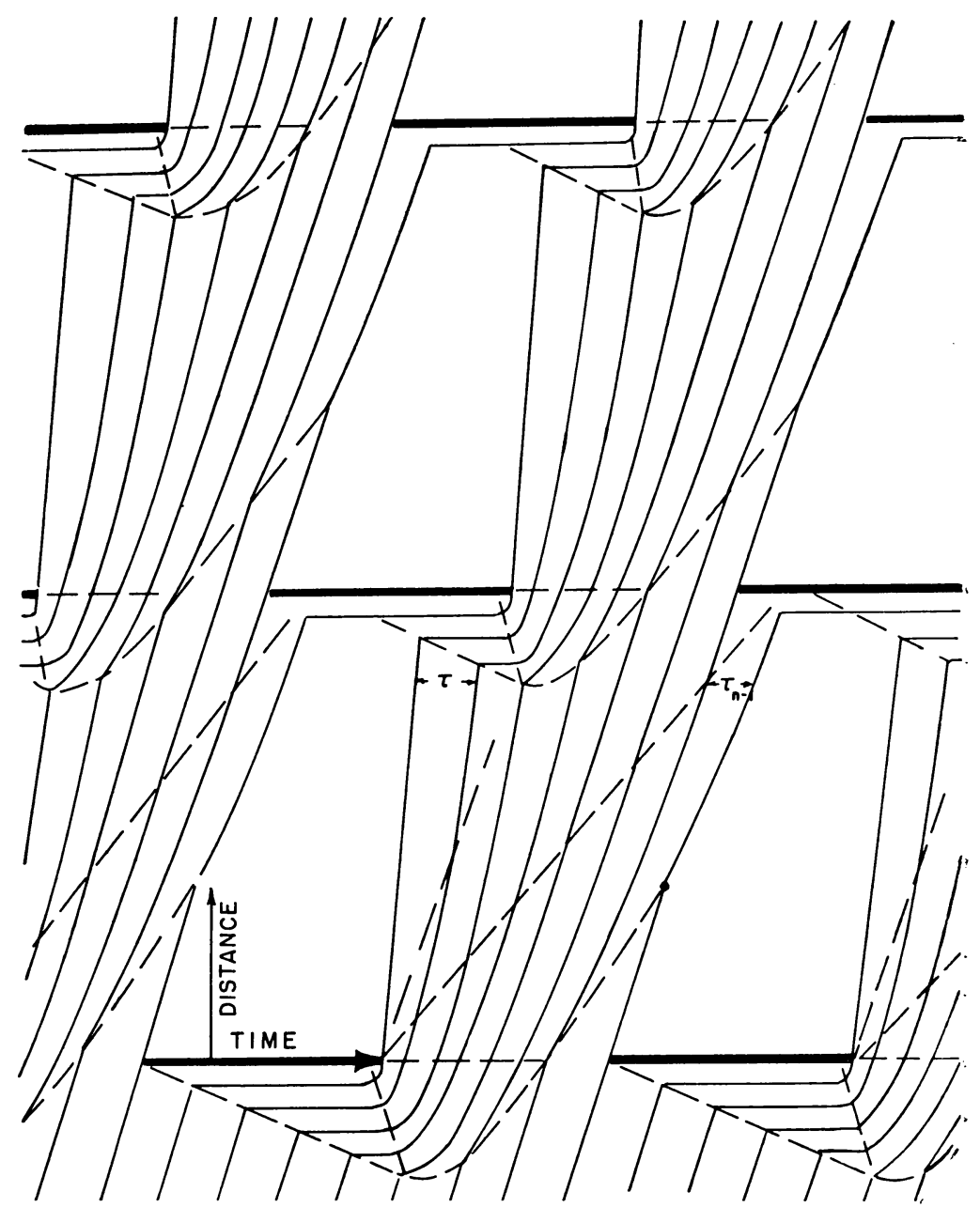

Fig. 2. 
of all $n$ arrival times at the third light by an amount $\tau_{n-1}$. We have

$$
D(t)=T-n t \text { for } 0<t<\tau_{n-1} \text {. }
$$

A lead car approaches a light at its free speed and each succeeding car is still trying to catch up with it. The velocity of any car is therefore less than its predecessor in the platoon and according to existing theories this implies that

$$
\tau_{1} \geq \tau_{2} \cdots \geq \tau_{n-1} .
$$

If the time interval between the last car of one platoon and the first car of the next platoon were less than $\tau_{1}$, the platoon would have merged. This possibility has not been considered in the above description and is rejected here. In this case there would not be much point in one trying to synchronize the lights anyway. If the platoons do not merge then

$$
T>\tau_{1}+\sum_{i=1}^{n-1} \tau_{i}>n \tau_{n-1}
$$

and so in (3) we still have $D(t)>0$.

As $t$ exceeds $\tau_{n-1}, D(t)$ again jumps by an amount $T$ because the $(n-1)$ th car is stopped by the light and shifts into the next platoon. For $\tau_{n-1}<t<\tau_{n-1}+\tau_{n-2}, D(t)$ again decreases by $n\left(t-\tau_{n-1}\right)$ until one is about to stop the $(n-2)$ th car. Thus

$$
D(t)=2 T-n t \text { for } \tau_{n-1}<t<\tau_{n-1}+\tau_{n-2}
$$

but still (5) gives $D(t)>0$.

The complete curve of delay per car per light, $D(t) / n$ vs $t$, is shown in Fig. 3. By continuing the above argument, we conclude that $D(t) / n$ jumps by $T / n$ at the times $\tau_{n-1}, \tau_{n-1}+\tau_{n-2}, \cdots$ but otherwise has unit negative slope. Since the light is cyclic with period $T, D(t)$ is a periodic function. It vanishes only at $t=0$ or equivalent times $t=k T$ with $k$ integer and otherwise $D(t)>0$ which means that $t=0$ is the optimal synchronization.

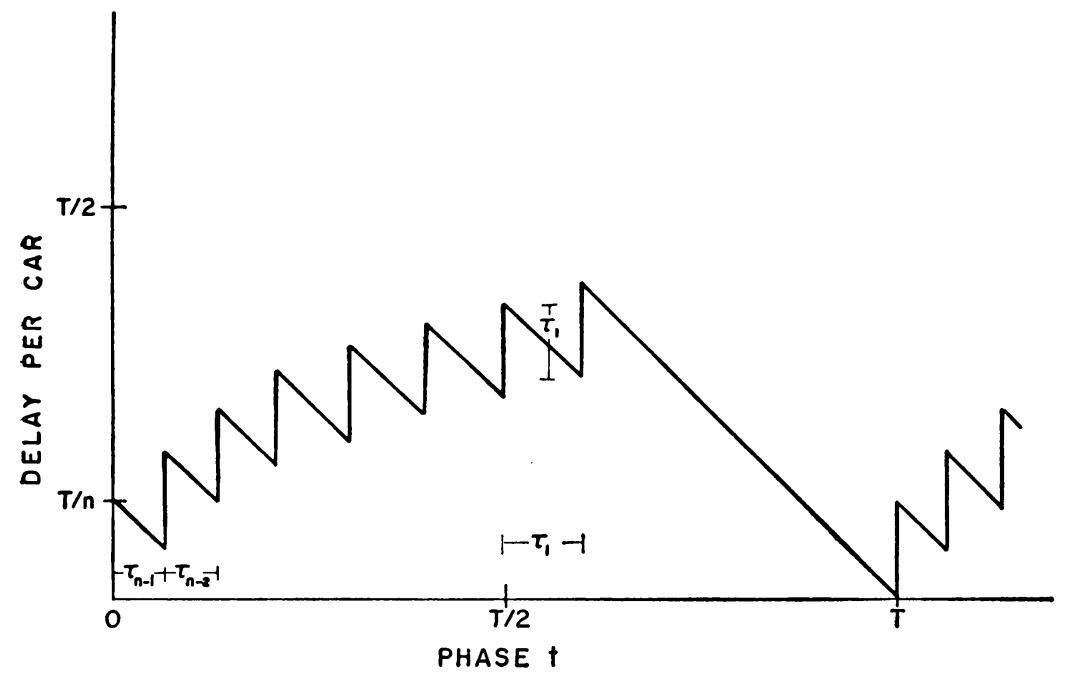

FIG. 3. 
This conclusion that $t=0$ is the optimal synchronization was based upon the assumption that the lead driver accelerates to his free speed independent of the synchronization. If we phase the lights as in Fig. 1, however, the lead driver, knowing that he will be stopped by the light if he drives at his free speed, may choose to drive slower. To him personally there is no reason to rush so he can wait. In so doing, however, he retards. the whole platoon and since the last car in the platoon barely makes the light at the higher speed, it now fails. If for the new speed the assumptions of the above theory still hold (duration of the platoon exceeds the green time, etc.) and one tries again to minimize the total delay for all cars one would have to retard the phase so that the last car still clears the light. With the retarded phase the lead car is, in effect, delayed more. The minimum delay is, therefore, still that of Fig. 1 in which the lead car travels at his. top speed.

Consequences. Here we shall consider briefly some consequences of (a) drivers who do not cooperate, (b) loss due to accelerations, (c) increases in the flow, and (d). fluctuations.

If one could establish the flow pattern shown in Fig. 1, it would be possible for a. single lead driver by traveling slower to arrive at the intersection just as the light turns. green. He perhaps realizes that by doing so he may be able to cross the intersection sooner than if he is stopped first and must accelerate again. Unfortunately this gain is bought at a high price to other drivers. Not only does this delay the last car in his platoon so that it. is stopped by the light but the next light cycle must try to pass $(n+1)$ cars. Furthermore the lead driver of the next platoon does not have the same opportunity of entering. the intersection just as the light turns since a car is already stopped in front of him. Even though some drivers will not cooperate, hopefully the majority will do what is in the best interest of everyone if they knew what was best.

Until now we have neglected the fact that a moving car can usually cross an intersection sooner than a stopped car. The red time of the light in Figs. 1 and 2 represents. an effective red time lasting until the first car can cross the intersection starting from whatever state of flow that happens to exist. It is true that if a lead driver decreases. his velocity and slows down the whole platoon, that the time headways of the platoon will decrease. If the velocity is decreased enough, one will eventually make the duration of the platoon less than the green time (contrary to the assumptions in the above theory). Under these conditions the whole platoon can pass the light without any car being stopped. and one might then be able to exploit any larger effective green time for cars that are not stopped. If the density of traffic is high, however, the effective loss in time that results. from forcing all cars to travel at a slower speed is certain to be larger than the gain in time one might achieve by eliminating the transient effects of starting. The proper way to eliminate the transient is not to lower the velocity of all cars between lights but to use some presignal [8] before the intersection that will allow the lead car to enter the intersection at moderate speed as soon as the light turns. This not only gets this car through the intersection sooner but also gives it a higher speed which will carry it to the next intersection sooner. The ideal situation is one for which the flow is essentially as shown in Fig. 1 but the queue is set back away from the intersection and one signals the lead car to start moving before the light turns green so he will enter the intersection at some. reasonable speed.

For the synchronization of Fig. 1, the phase of the light depends among other things upon the flow rate $q$ or equivalently $n$. If we change $n$ to $n+1$ in Fig. 1, the addition of 
an $(n+1)$ th car should not change the times $\tau_{1}, \cdots, \tau_{n-1}$ between the first $n$ cars. The $(n+1)$ th car arrives at a time $\tau_{n}$ after the $n$th car in Fig. 1 . To optimize the phase in the presence of the extra car, we must delay the light by $\tau_{n}$ so that the new car barely clears the light. All cars will then move in phase with the new synchronization so the extra car causes a delay of $\tau_{n}$ per light for each of the original $n$ cars. As the flow increases the lights are in effect synchronized for a slower velocity. If intersections are not too close together, the addition of a time $\tau_{n}$ to the journey time from one intersection to the next may not seem very important from the point of view of an individual driver. But this loss is suffered by every car, so that the total loss per cycle to the original $n$ cars is $n \tau_{n}$ which is comparable with (perhaps half) a cycle time. If a driver's time is worth as much as a policeman's time, the cost of increasing the flow from $n$ to $n+1$ cars per cycle is comparable with the cost of having a policeman at the intersection.

The theory described here was based upon an idealized model in which all drivers behave in exactly the same way. In effect we have replaced the average behavior of many different drivers by the behavior of many identical average drivers. We have also assumed that we had $n$ cars in every platoon with no fluctuations.

A detailed analysis of statistical fluctuations for a theory of this type would be quite tedious even for simple stochastic models. The important thing to ask is whether or not the synchronization scheme leads to such a highly organized flow pattern that small fluctuations in behavior would give rise to large deviations from the flow pattern described here. Is the flow reasonably stable?

One can easily investigate qualitatively what would happen if one should add an extra car to some platoon, some lead driver drives too slow or too fast, or any other obvious deviation from the ideal pattern considered above. In every case one concludes that these deviations produce only transient disturbances provided that the average flow rate does not exceed the capacity of the intersections. Figure 2 also shows that small deviations from the optimal synchronization give only relatively small increases in delay. Furthermore the synchronization has a tendency to cut cars off long platoons and shift them into subsequent platoons until they can be absorbed by a short one. We conclude that the flow pattern of Fig. 1 is quite stable and even though fluctuations may be important in describing the details of the flow pattern they are not likely to alter the qualitative conclusions described here.

Two-way traffic. One unpleasant feature of two way traffic is that the optimal synchronization of the lights for one direction of traffic is almost certainly not the optimal synchronization for the opposing traffic. One possible criterion for selection of the synchronization is to minimize the total delay for all cars in both directions.

If we assume that the flows in both directions are so large that the theory described above is applicable to both directions of traffic, the best pairwise synchronization of lights again gives the best global synchronization. If we number the intersections consecutively, then the arrival pattern at the $j$ th intersection of cars coming from the $(j-1)$ th intersection depends only upon the relative phase of these two lights but is independent of the phase of other lights. Similarly the delays at the $(j-1)$ th intersection of cars coming from the $j$ th intersection depends only upon the relative phase of these lights. The optimal synchronization of all lights thus results when one selects the relative phase of the $(j-1)$ th and $j$ th lights to minimize the sum of delays for cars at the $j$ th light which have come from the $(j-1)$ th lights and for cars at the $(j-1)$ th light which have come from the $j$ th light. 
Figure 3 gives the delay per car for one direction of traffic as a function of the phase. We need only multiply this by $n$ to obtain the total delay for these cars per cycle. If Fig. 3 represents the delay for traffic moving from the $(j-1)$ th to $j$ th intersections, the phase $t$ of Fig. 3 is the time which elapses from the moment the light turns red for these cars at intersection $j$ until the last car in the approaching platoon arrives. If we draw an analogous figure for the opposing traffic as a function of a phase $t^{\prime}$ defined in the corresponding way for the opposing stream, then $t^{\prime}$ is the time elapsed after the light turns red for these cars at intersection $(j-1)$ until the last car of this platoon arrives.

Although $t$ and $t^{\prime}$ both measure the relative phase of the $(j-1)$ th and $j$ th lights they differ in that an increase of $t$ gives a decrease in $t^{\prime}$ and furthermore $t=0$ will not generally correspond to $t^{\prime}=0$. If we wish to represent all delays as a function of the same phase variable, for example $t$, we must draw the graph of delays to the reverse traffic as a function of $t$ and then add the two delay curves. To do this one need only know the value of $t^{\prime}$ corresponding to one value of $t$ since the correspondence for all other $t$ can be found by subtracting from $t$ whatever one adds to $t^{\prime}$. The value of $t^{\prime}$ corresponding to a given $t$ depends upon the trajectories of the cars and can best be found experimentally. One simply observes $t$ at the $j$ th intersection and for the same setting of the lights the value of $t^{\prime}$ at the $(j-1)$ th light.

In Fig. 4 the lower solid line graph is a reproduction of Fig. 3 with the delays multiplied by $n$. Here $n=8$. The broken line curve is a possible delay curve for the opposing traffic for $n^{\prime}=7$. The upper solid line graph is the sum of these. Whereas in Fig. 3, the slope of the graph was equal to one at every continuity point, the slopes in Fig. 4 are $n$, $n^{\prime}$, and $n-n^{\prime}$ respectively. In Fig. 3 all discontinuities were jumps by $T / n$ whereas in Fig. 4 the jumps are either up or down but always by an amount $T$ regardless of direction.

In the example of Fig. 4, the optimal synchronization is at $t=0$ or $T$, the optimal phase for the forward traffic alone. If the broken line graph were shifted slightly to the right, however, the narrow dip in the total delay curve at $T$ would be annihilated and the

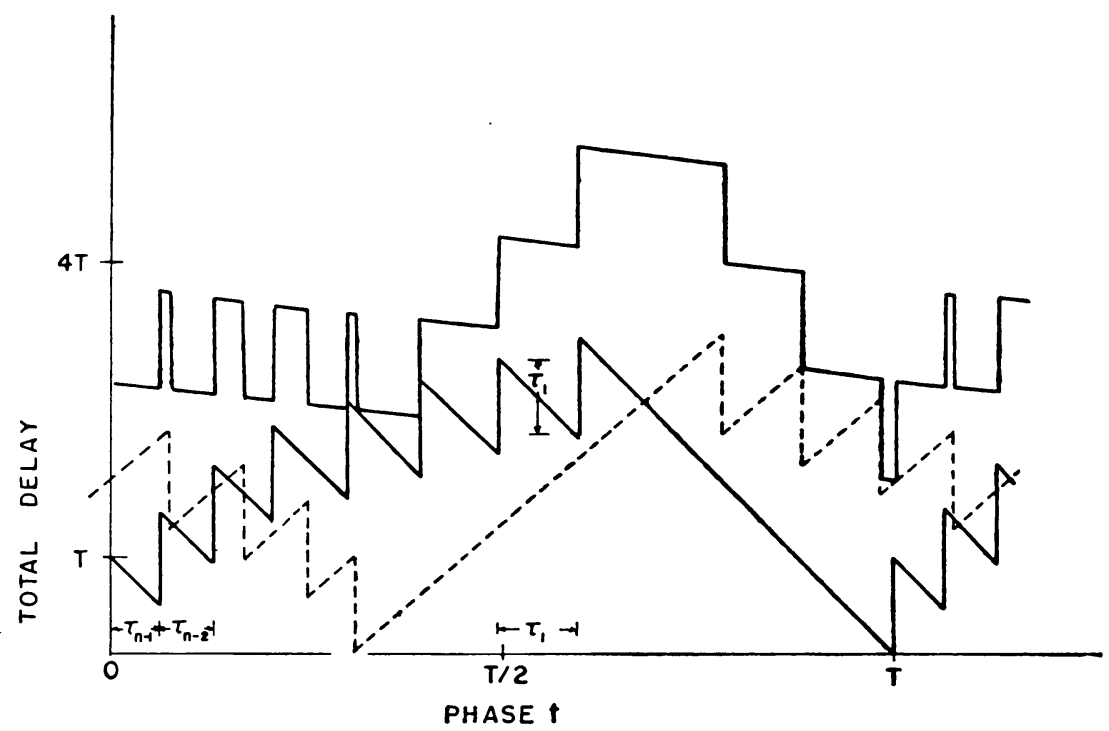

Fig. 4. 
minimum point would shift to a point near where the opposing traffic has minimum delay.

Since we are neglecting statistical fluctuations, the details of these graphs cannot be taken too seriously, but one can detect a tendency for the optimal synchronization often to be near that which is optimal for the direction of traffic with the lower flow rate (contrary to Fig. 4). This odd phenomena arises because the penalty when one stops an extra car at the tail of a platoon is $T$ (the jumps in Fig. 4), the same for both directions of traffic. For the forward traffic we would like to advance the light to help the lead cars if it were not for the fact that this stops cars at the tail of the platoon. If by advancing the phase, however, we can compensate for each car stopped at the tail of the platoon for the forward stream by a reduction in the number stopped in the opposing stream, the net effect is a gain for $n>n^{\prime}$. It is likely that one can balance these gains and losses for cars at the tail of the platoons until the light is advanced to a point where no cars are stopped at the tail of the platoon in the opposing stream. This is the phase which is optimal for the opposing stream alone.

We shall not pursue this further since a complete description of the optimal phase as a function of all the time headways is tedious.

\section{REFERENCES}

1. G. F. Newell, The flow of highway traffic through a sequence of synchronized traffic signals, Opns. Res. 8 390-405 (1960)

2. M. J. Lighthill and G. B. Whitham, On kinematic waves II: A theory of traffic flow on long crowded roads, Proc. Royal Soc. A229, 317-345 (1955)

3. P. I. Richards, Shock waves on the highway, Opns. Res. 4, 42-51 (1956)

4. R. F. Chandler, R. Herman, and E. W. Montroll, Traffic dynamics: Studies in car following, Opns. Res. 6, 165-184 (1958)

5. E. Kometani and T. Sasaki, On the stability of traffic flow, J. Opns. Res. Japan 2, 11-26 (1958)

6. R. Herman, E. W. Montroll, R. B. Potts, and R. W. Rothery, Traffic dynamics: analysis of stability in car following, Opns. Res. 7, 86-106 (1959)

7. G. F. Newell, Nonlinear effects in the dynamics of car following, Opns. Res. 9, 209-229 (1961)

8. W. von Stein, Traffic flow with pre-signals and the signal funnel, Theory of Traffic Flow. R. Herman ed., Elsevier, 1961, pp. 28-56. 